outcome for this teenager. Longterm surveillance for tumour detection is recommended in autoimmune encephalitis.

\section{G66(P) INTERESTING CASES OF PYRIDOXINE DEPENDENT EPILEPSY IN LATE CHILDHOOD}

S Jain, E Wassmer. Neurology, Birmingham Children's Hospital, Birmingham, UK

\subsection{6/archdischild-2015-308599.65}

We report cases of two children with intractable epilepsy who responded to treatment with pyridoxine in late childhood.

Case details Case 1: A 14 year old girl born following an uneventful pregnancy to non consanguinous parents. She has global developmental delay. She developed epilepsy at around 2 years of age. Her extensive investigations including an MRI of brain were normal. She had various types of seizures and these were refractory to antiepileptic medications. A trial of pyridoxine at around 13 years of age surprisingly had a very good effect on her seizure control. We are currently awaiting the result of urinary L- $\alpha$-aminoadipic semialdehyde (AASA).

Case 2: A 4 year old girl born following normal pregnancy developed infantile spasms at 4 months of age. She was treated with numerous courses of steroids with suboptimal response. Extended investigations including the MRI brain were normal. She eventually developed refractory epilepsy with cognitive decline. A trial of pyridoxine at 3 years of age achieved good control of epilepsy and improved her cognition as well. The urinary AASA was negative and the result of PNPO gene mutation is awaited.

Discussion Pyridoxine dependent epilepsy is a syndrome that usually presents with neonatal intractable seizures. It may present later in infancy or early childhood. A variety of seizure types are reported, the most common being generalised tonic clonic seizures which progress to status epilepticus. Urinary AASA is usually elevated and the genetic testing is available for this condition now. Our cases responded to the empirical trial of Pyridoxine at a much later age.

Conclusion We recommend testing for pyridoxine deficiency and therapeutic trial with pyridoxine in children with refractory epilepsy in all the age groups. Early detection and treatment of this condition not only achieves good seizure control but can possibly improve developmental outcome as well.

\section{G67(P) CHILDHOOD PARKINSONISM: A RARE COMPLICATION OF HYPOXIC BRAIN INJURY}

${ }^{1} \mathrm{M}$ Ramphul, 'A Joshi, ' $\mathrm{A}$ Maw. 'Paediatrics, Luton and Dunstable University Hospital, Luton, UK; ${ }^{2}$ Paediatric Neurology, Cambridge University Hospital, Cambridge, UK

\subsection{6/archdischild-2015-308599.66}

Case report Child A is a 13-year old boy with a history of poor adherence to his asthma treatment.

He was admitted to hospital with an acute severe exacerbation of asthma, which needed escalation of treatment to intravenous bronchodilators. Despite intensive treatment, he went on to have a cardio-respiratory arrest, necessitating five minutes of cardiopulmonary resuscitation and adrenaline prior to return of his circulation.

Child A spent a week on PICU, after which he underwent neurological rehabilitation on the ward, involving a multidisciplinary team. MRI of his brain confirmed severe hypoxic brain

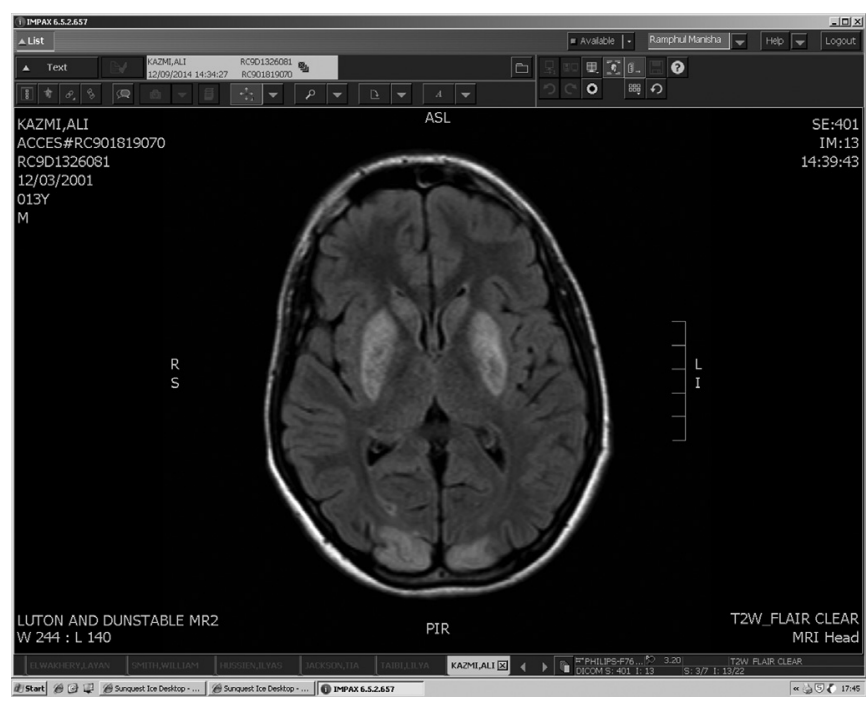

Abstract G67(P) Figure 1

injury with infarction within the basal ganglia and occipital lobe (Figure 1). Similar to other children with acquired brain injury, he demonstrated 4-limb spasticity, as well as dystonic posturing.

In addition to this, he also displayed Parkinsonian features, which is a rare complication of brain injury. He had a resting tremor, bradykinesia, rigidity and a shuffling gait, with difficulty in turning around. He displayed hypomimic facies, along with a monotonous speech. The above symptoms responded well to cocareldopa.

Discussion Poor compliance with asthma medication in children remains a significant problem with major health implications. Our case sustained a severe brain injury as a result of this. Unlike in adults where Parkinsonism is common, this condition is rare in children and easy to miss. The commonest cause of Parkinsonism is the loss of dopaminergic neurones in the substantia niagra of the basal ganglia. Child A's symptoms resolved with co-careldopa which increases dopamine levels in the brain.

We have not come across a similar paediatric case during our literature review. Paediatricians need to be vigilant to identify Parkinsonian features in children with brain injury. These typical signs become apparent to the clinician who is clearly looking out for them. Making this diagnosis correctly is important so that we can prescribe a specific anti-Parkinsonian medication, rather than make the child go through repeated failed trials with incorrect drugs. There are different classes of medication for Parkinsonism and treatment choices are largely based on data from adults.

Recognising this movement disorder is hence vital to support the rehabilitation process and optimise recovery.

\section{G68(P) A RETROSPECTIVE REVIEW OF EPILEPSY RELATED ADMISSIONS TO THE PAEDIATRIC DEPARTMENT AT A DISTRICT GENERAL NHS TRUST, ENGLAND}

O Aziz, TKW Ramcharan, D Gandhi. Paediatrics, Sandwell and West Birmingham NHS Trust, West Bromwich, UK

\subsection{6/archdischild-2015-308599.67}

Aims Child and Maternal Health (ChiMat) Observatory Data indicate our institution has higher Paediatric Emergency Epilepsy admissions and length of stay, compared to other PCTs in 